\title{
A GENERALIZATION OF FEIT'S THEOREM
}

\author{
BY
}

\section{J. H. LINDSEY II}

\begin{abstract}
This paper is part of a doctoral thesis at Harvard University. The title of the thesis is Finite linear groups in six variables.

Using the methods of this paper, I believe that I can prove that if $p$ is a prime greater than five with $p \equiv-1(\bmod 4)$, and $G$ is a finite group with faithful complex representation of degree smaller than $4 p / 3$ for $p>7$ and degree smaller than 9 for $p=7$, then $G$ has a normal $p$-subgroup of index in $G$ divisible at most by $p^{2}$. These methods are particularly effective when there is nontrivial intersection of $p$-Sylow subgroups. In fact, if the current work people are doing on the trivial intersection case can be extended, it should be possible to show that, for $p$ a prime and $G$ a finite group with a faithful complex representation of degree less than $3(p-1) / 2, G$ has a normal $p$-subgroup of index in $G$ divisible at most by $p^{2}$. (It may be possible to show that the index is divisible at most by $p$ if the representation is primitive and has degree unequal to $p$.)
\end{abstract}

Introduction. In this paper all representations are assumed to be over $C$, the complex numbers. We use standard mathematical notation without comment. If $X$ is a representation of the group $G$ on the vector space $V$, we call a subspace $U$ of $V$ a homogeneous space for $G$ if $U$ is invariant for $G$ and $U$ is maximal with the property that the irreducible constituents of the representation of $G$ on $U$ are equivalent. The representation $X$ is called quasiprimitive if it is irreducible, and for all normal subgroups $N$ of $G, X \mid N$ has just the homogeneous subspace $V$. For $x \in G$ and $\gamma \in C, C_{V}\left(\gamma^{-1} x\right)=\langle v \mid v \in V, X(x) v=\gamma v\rangle$ and for $H \subset G, C_{V}(H)$ $=\bigcap_{h \in H} C_{V}(h)$. The term $i_{\Pi}$ is defined in the following theorem. This theorem generalizes the theorem in [5]. Equality is allowed by $C$.

THEOREM. Let $\Pi$ be a set of primes and let $X$ be a faithful representation on the vector space $V$ of the finite group $G$ of degree $n$ over the complex numbers. Define $i_{\Pi}(G)=|G|_{\Pi} /\left|O_{\Pi}(G)\right|$. Assume that $p \geqq n+1, p \geqq 7$ for all $p \in \Pi$. Assume that $G$ has a П-Sylow subgroup, $H$. Then either:

I. $i_{\Pi}(G)$ is not composite.

II. $X$ is imprimitive or reducible on the spaces $V_{1}$ and $V_{2}$ of dimension $n / 2$ where $V=V_{1} \oplus V_{2}$. Also, $n+1=p \in \Pi$ for some $p$. There exists a normal subgroup $M$ of

Received by the editors August 18, 1969 and, in revised form, October 30, 1969.

AMS 1969 subject classifications. Primary 2072, 2075, 2080.

Key words and phrases. Quasiprimitive, homogeneous space, $\operatorname{PSL}(2, p)$.

Copyright (C) 1971, American Mathematical Society 
$G$ of index 1 or 2 having the $V_{i}$ as invariant, irreducible subspaces. There exist subgroups $T_{i}$ of $M$ with $M=Z(M)\left(T_{1} \times T_{2}\right) ; C_{V}\left(T_{i}\right)=V_{i}$; and

$$
\begin{aligned}
T_{i} & \simeq \operatorname{PSL}(2, p), \quad p \equiv-1(\bmod 4), \\
& \simeq S L(2, p), \quad p \equiv 1(\bmod 4), \quad \text { for } i=1,2 .
\end{aligned}
$$

The theorem generalizes [5] when $n+1 \in \Pi$. When $n+1 \notin \Pi$, an abelian $\Pi$ Sylow subgroup of $G$ was guaranteed by Blichfeldt. When $n+1 \in \Pi$, the existence of a П-Sylow subgroup must be assumed (such a group is abelian by Lemma 7). For example $S L(2,13)$ has a representation of degree 6 , but has no subgroup of order (7)(13). When $n+1 \notin \Pi$, our proof uses only Lemmas 1 and 2 and furnishes a short proof of Feit's Theorem. Furthermore, when for $p \in \Pi, p \geqq 2 n+1$, we may use our proof to prove that $i_{\Pi}(G)=1$ or $p=2 n+1 \in \Pi$ for some $p$ and $G / Z(G)$ $\simeq \operatorname{PSL}(2, p)$, the result of [6], by induction on $n$. Here, only Lemma 1 is needed. Also, (E) and $(\mathrm{H})$ follow immediately from the stronger induction hypothesis that $i_{\Pi}\left(G_{0}\right)=1$ when $G_{0}$ has a faithful representation of degree $n_{0}<n$. Then only steps (A), (B), (C), (F), (I), and (J) are needed to complete the proof, since [2] can be applied if $|G|_{\Pi}$ is prime.

LEMMA 1. If $G$ is a finite group and $\Pi$ is a set of primes, define $i_{\Pi}(G)=|G|_{\Pi} /\left|O_{\Pi}(G)\right|$. Then if $H$ is a homomorphic image or a subgroup of $G, i_{\Pi}(H) \mid i_{\Pi}(G)$. Furthermore, if $K$ and $L$ are finite groups, $i_{\Pi}(K \times L) \mid\left(i_{\Pi}(K)\right)\left(i_{\Pi}(L)\right)$. Finally, $i_{\Pi}(G)=i_{\Pi}(G / Z(G))$.

Proof. If $\alpha$ is a homomorphism from $G$ onto $H$ with kernel $K$, then $\alpha\left(O_{\Pi}(G)\right)$ $\subset O_{\mathrm{\Pi}}(H)$ and

$$
|H|_{\Pi} /\left|\alpha\left(O_{\Pi}(G)\right)\right|=\left[|G|_{\Pi} /|K|_{\Pi}\right] /\left[\left|O_{\Pi}(G)\right| /\left|K \cap O_{\Pi}(G)\right|\right]
$$

which divides $|G|_{\Pi} /\left|O_{\Pi}(G)\right|$. If $H \subset G$ and $\beta$ is the natural homomorphism from $G$ to $G / O_{\Pi}(G)$, then $H \cap O_{\Pi}(G) \subset O_{\Pi}(H)$ and $H / H \cap O_{\Pi}(G) \simeq \beta(H) \subset G / O_{\Pi}(G)$. The middle statement of Lemma 1 follows from $O_{\Pi}(K) \times O_{\Pi}(L) \subset O_{\Pi}(K \times L)$. We have already shown that $i_{\Pi}(G / Z(G)) \mid i_{\Pi}(G)$. Let $\gamma$ be the natural homomorphism of $G$ into $G / Z(G)$. Let $M=\gamma^{-1}\left(O_{\Pi}(G / Z(G))\right)$. Then $Z(G)=[Z(G)]_{\Pi} \times[Z(G)]_{\Pi}$, where $[Z(G)]_{\Pi^{\prime}}$ is characteristic in $Z(G)$ and is a normal $\Pi^{\prime}$-Sylow of $M$. By SchurZassenhaus, there exists $N$, a $\Pi$-Sylow subgroup of $M$. As $M=N \times[Z(G)]_{\Pi^{\prime}}, N$ is characteristic in $M$ which is normal in $G$. Therefore, $N \subset O_{\Pi}(G)$. As $|G|_{\Pi} /|N|=$ $|G / M|_{\Pi}=|G / Z(G)|_{\Pi} /\left|O_{\Pi}(G / Z(G))\right|$, this concludes the proof of Lemma 1 .

LEMMA 2. Let $X$ be a faithful irreducible representation of a finite group $G$ which affords the character $\chi$. Let $p \geqq 5$ be a prime. Let $H=\left(O^{p^{\prime}}(G)\right)^{\prime}$ and let $P$ be a p-Sylow subgroup of $H$. Assume that $i_{p}(G)=p$ and $n=\chi(1)<p-1$. Then

(i) $X$ is primitive, $n \geqq(p-1) / 2, p \||H|, i_{p}(H)=p$ and $O^{p^{\prime}}(G) \subseteq H Z(G)$.

(ii) $X \mid H$ is irreducible.

(iii) $\chi \mid P$ is the sum of distinct linear characters. The principal character of $P$ is contained in this sum if and only if $n>(p-1) / 2$.

(iv) If $x$ is a p-element of $G$ then either $X(x)$ is scalar or has distinct eigenvalues. 
Proof. By [6], $i_{p}(G) \neq 1$ implies that $n \geqq(p-1) / 2$. If $X$ is imprimitive on the spaces $V_{1}, \ldots, V_{m}$, let $K$ be the subgroup of $G$ fixing the $V_{i}$. As $\operatorname{dim} V_{i}=n / m$ $<(p-1) / 2$, the constituent $X_{i}(K)$ of $X(K)$ acting on $V_{i}$ satisfies $i_{p}\left(X_{i}(K)\right)=1$ for $i=1, \ldots, m$. As $|G / K| \mid m$ ! and $O_{p}(K)$ is characteristic in $K$ which is normal in $G$, $O_{p}(K)$ is a normal $p$-Sylow subgroup of $G$, a contradiction.

Using Blichfeldt's method of replacing a generator $X(x)$ by

$$
Y(y)=[\operatorname{det} \chi(x)]^{-1 / n} X(x),
$$

one may find a finite group $L$ with a unimodular representation $Y$ of degree $n$ with $Y(L)(Z G L(n, C))=X(G)(Z G L(n, C))$. Then $Y$ is primitive. Furthermore,

$$
L / Z(L) \simeq Y(L)(Z G L(n, C)) / Z G L(n, C)=X(G)(Z G L(n, C)) / Z G L(n, C) \simeq G / Z(G)
$$

By Lemma $1, i_{p}(L)=i_{p}(G)=p$. As $n<p-1, O_{p}(L)$ is abelian by Lemma 7. As $O_{p}(L)$ is normal in $L, Y(L)$ permutes the homogeneous spaces of $Y \mid O_{p}(L)$ (the sums of spaces on which identical constituents of $Y \mid O_{p}(L)$ act). Therefore, all constituents of $Y \mid O_{p}(L)$ are identical and $Y\left(O_{p}(L)\right)$ consists of scalars of the form $\alpha I_{n}$. Then $\alpha$ is a $p$ th root of unity for some $t$ and $\alpha^{n}=\operatorname{det}\left(\alpha I_{n}\right)=1$. As $n<p-1$, $\alpha=1$ and $O_{p}(L)=\langle 1\rangle$. Therefore, $p \||L|$. Then Lemma 3.1 of [5] applies to $L$ and implies that $\left(O^{p^{\prime}}(L)\right)^{\prime}=O^{p^{\prime}}(L)$. If $x$ is a $p$-element in $G$, then there exists $z \in Z G L(n, C)$ with $z X(x)=Y(y)$ for some $y$ in $L$. As $\langle X(x), Y(y)\rangle \subset\langle z, X(x)\rangle$, an abelian subgroup, $z=Y(y)^{-1} X(x)$ is of finite order. Then $[z]_{p} X(x)=[z X(x)]_{p}$ $=[Y(y)]_{p}$ is a power of $Y(y)$, and replacing $z$ by $[z]_{p}$, we may take $y$ to be a p-element. This and the symmetric argument show that $X\left(O^{p^{\prime}}(G)\right) Z G L(n, C)$ $=Y\left(O^{p^{\prime}}(L)\right) Z G L(n, C)$. Then $X(H)=\left(X\left(O^{p^{\prime}}(G)\right)\right)^{\prime}=\left(Y\left(O^{p^{\prime}}(L)\right)\right)^{\prime}=Y\left(O^{p^{\prime}}(L)\right)$. Therefore, $p \||H|, \quad i_{p}(H)=p, \quad X\left(O^{p^{\prime}}(G)\right) \subset Y\left(O^{p^{\prime}}(L)\right) Z G L(n, C)=X(H) Z G L(n, C)$, and $O^{p^{\prime}}(G) \subset H Z(G)$. By [6], irreducible constituents $X_{i}(H)$ of $X \mid H$ with $i_{p}\left(X_{i}(H)\right)=p$ have degree $\geqq(p-1) / 2$. As $n<p-1$, there is at most one such constituent. By Lemma 1 , there is at least one such constituent. If $W$ is the space on which this constituent acts and $x \in G$, then $H=x H x^{-1}$ has $x W$ as an irreducible invariant space for some constituent $U$ of $X \mid x H x^{-1}$ and $i_{p}\left(U\left(x H x^{-1}\right)\right)=p$. Therefore, $x W$ $=W$ and by irreducibility of $X, \operatorname{dim} W=n$ and $X \mid H$ is irreducible. The statement in Lemma 2 about $\chi \mid P$ follows from Lemma 3.1 of [5] applied to $Y\left(O^{p^{\prime}}(L)\right)=X(H)$. The final statement of Lemma 2 follows from our previous step where for $x$ a $p$-element in $G$ there exist $y \in L$ and $z \in Z G L(n, C)$ with $[z]_{p} X(x)=[Y(y)]_{p}$, which is $I_{n}$ or has distinct eigenvalues.

The remaining lemmas are needed in the proof of our theorem only in the case where we have a proper generalization of Feit's Theorem $(n+1 \in \Pi)$. Some of the proofs of these lemmas require Feit's Theorem.

Lemma 3. Let $X$ be a faithful, irreducible representation of a finite group $G$ of degree $(p-1) / 2$ for $p$, a prime greater or equal to 5 . Suppose $G$ does not have a normal $p$-Sylow subgroup. Then $G=G^{\prime} Z(G)$ where $G^{\prime} \simeq P S L(2, p)$ if $(p-1) / 2$ is odd 
and $G^{\prime} \simeq S L(2, p)$ if $(p-1) / 2$ is even. There are exactly two distinct irreducible representations of $G^{\prime}$ of degree $(p-1) / 2$.

Proof. As in the proof of Lemma 2, there exists a finite group $L$ with a faithful, unimodular $(p-1) / 2$-dimensional representation $Y$ with $Y(L) Z G L(n, C)=$ $X(G) Z G L(n, C)$ and the following properties: $i_{p}(L)=p, p \||L|$. By [2], $L / Z(L)$ $\simeq P S L(2, p)$. Then $(X(G))^{\prime}=(Y(L))^{\prime} \simeq P S L(2, p)$ or $S L(2, p)$ by [11]. Furthermore,

$$
(X(G))^{\prime} Z G L(n, C)=(Y(L))^{\prime} Z G L(n, C)=Y(L) Z G L(n, C)=X(G) Z G L(n, C) .
$$

Therefore, $G=G^{\prime} Z(G)$. The remainder of the lemma follows from the classification in [11] of projective representations of $\operatorname{PSL}(2, p)$.

Lemma 4. Let $L$ be a subgroup of a finite group $G$ and $i_{p}(L)=i_{p}(G)=p$ for $a$ prime $p \geqq 5$. Let $X$ be a faithful, irreducible representation over $C$ of $G$ of degree $n<p-1$. Let $Y$ be the unique constituent of $X \mid L$ which is irreducible and satisfies $i_{p}(Y(L))=p$. Let $m=\operatorname{deg} Y$. Then $m=n$ or $m=n-1=(p-1) / 2 .($ Actually, by [12], $m=n$.)

Proof. Let $X \mid L=W \oplus Y$ for some constituent $W$ of $Y \mid L$. Then $i_{p}(W(L))=1$, by Lemma 1. By [1], $O^{p^{\prime}}(W(L))=O_{p}(W(L))$ is abelian and $\left(O^{p^{\prime}}(W(L))\right)^{\prime}=\langle 1\rangle$. For any $p$-element $M$ in $Y(L)$ we may find $x \in L$ with $Y(x)=M$. Then $Y\left([x]_{p}\right)=[Y(x)]_{p}$ and we may take $x$ to be a $p$-element. Therefore, $Y\left(O^{p^{\prime}}(L)\right)=O^{p^{\prime}}(Y(L))$. Then by Lemma 2, we may find $y \in\left(O^{p^{\prime}}(L)\right)^{\prime}$ with $Y(y)$ having order $p$ and $m$ distinct eigenvalues, one of which is 1 if and only if $m \geqq(p-1) / 2$. Also, $W(y) \in\left(O^{p^{\prime}}(W(L))\right)^{\prime}$ $=\langle 1\rangle$. Applying Lemma 2 to $y \in G$ and the representation $X$, we see that $X(y)$ has distinct eigenvalues. This implies the conclusion of Lemma 4.

LEMMA 5. Let $X$ be a faithful, reducible representation of the finite group $G$. Let $X=X_{1} \oplus X_{2}$ where $\operatorname{deg} X_{1} \leqq(p+1) / 2$, deg $X_{2}<p-1, p$ is a prime greater than 4 , $X_{i}$ is irreducible and $i_{p}\left(X_{i}(G)\right)=p$ for $i=1,2$; and $i_{p}(G)>p$. Then there exists $x \in\left(O^{p^{\prime}}(G)\right)^{\prime} \cap \operatorname{ker} X_{2}$ of order $p$ with $X_{1}(x)$ having exactly $(p-1) / 2$ eigenvalues unequal to 1. Furthermore, if $\operatorname{deg} X_{1}=\operatorname{deg} X_{2}=(p-1) / 2$, then $G=Z(G)\left(G_{1} \times G_{2}\right)$ where for $i=1,2, G_{i} \subset \operatorname{ker} X_{i} \cap O^{p^{\prime}}(G)^{\prime}$ and $G_{i} \simeq P S L(2, p)$ if $(p-1) / 2$ is odd, $G_{i} \simeq S L(2, p)$ if $(p-1) / 2$ is even.

Proof. Let $\alpha$ be the natural homomorphism $G \rightarrow Y_{1}(G) \times Y_{2}(G)$ where $Y_{i}(G)$ $=X_{i}(G) / Z\left(X_{i}(G)\right)$ for $i=1,2$. Then $\operatorname{ker} \alpha=Z(G)$ and by Lemma $1, i_{p}(\alpha(G))=i_{p}(G)$ $>p$. By Lemma 2,p $\|\left|Y_{i}(G)\right|$ for $i=1,2$. Then $\left.p^{2}|| \alpha(G)\right|_{p}=\left|Y_{2}(G)\right|_{p}\left|\left(\operatorname{ker} Y_{2}\right) / Z(G)\right|_{p}$ and $p|| \operatorname{ker} Y_{2} / Z(G) \mid$. Let $K=\operatorname{ker} Y_{2}$. Then $K \triangleleft G, \quad Y_{1}(K) \triangleleft Y_{1}(G)$, and $X_{2}(K)$ $\subset Z\left(X_{2}(K)\right)$. Since $p|| Y_{1}(K) \mid$, by Lemma $2, O^{p^{\prime}}\left(Y_{1}(G)\right) \subset Y_{1}(K)$ and $O^{p^{\prime}}\left(X_{1}(G)\right)$ $\subset X_{1}(K) Z\left(X_{1}(G)\right)$. Then $K^{\prime} \subset \operatorname{ker} X_{2}$ and $X_{1}\left(K^{\prime}\right) \supset\left(O^{p^{\prime}}\left(X_{1}(G)\right)\right)^{\prime}$ which by Lemma 2 contains an element $x$ of order $p$ with exactly $(p-1) / 2$ eigenvalues unequal to 1 . If $\operatorname{deg} X_{1}=(p-1) / 2$, then by Lemma 3, $\left(O^{p^{\prime}}\left(X_{1}(G)\right)\right)^{\prime} \simeq(P) S L(2, p)$ and $X_{1}(G)$ $=\left(O^{p^{\prime}}\left(X_{1}(G)\right)\right)^{\prime} Z\left(X_{1}(G)\right)$. Defining $G_{2}=K^{\prime}$ and reversing the roles of $X_{1}$ and $X_{2}$ for $\operatorname{deg} X_{1}=\operatorname{deg} X_{2}=(p-1) / 2$ finishes the proof of Lemma 5. 
LEMMA 6. Let $p$ be a prime $\geqq 5$ and $G$ be a finite group with a faithful representation $X$ of degree $n=p-1$. Let $L \subset G$ with $L / Z(L) \simeq P S L(2, p),(|Z(L)|, p)=1$, and $X \mid L$ $=X_{1} \otimes I_{2}$ with $\operatorname{deg} X_{1}=(p-1) / 2$. Let $P$ be a p-Sylow subgroup of $L$ and $A$ be an abelian subgroup of $G$ with $P \subset A$. If $A Z(G)($ or $A)$ is a trivial intersection set of $G / Z(G)$ (or $G)$, then $X \mid\langle A, L\rangle$ is reducible.

Proof. By [11], $X_{1} \mid N_{L}(P)$ is irreducible. By [4, Lemma 51.2], $C_{G L(n, C)}\left(X\left(N_{L}(P)\right)\right)$ $=I_{n / 2} \otimes G L(2, C)=C_{G L(n, C)}(X(L))$. Therefore, $X \mid N_{L}(P)$ and $X \mid L$ have the same invariant subspaces. As $P \subset A, P \notin Z(G)$, and $A Z(G)$ (or $A$ ) is a T. I. S. of $G / Z(G)$ (or $G$ ) it follows that $N_{L}(P) \subset N(A Z(G)$ ). Furthermore,

$$
\left|\left\langle N_{L}(P), A Z(G)\right\rangle\right| A Z(G)|\leqq| N_{L}(P) / P \mid=(p-1) / 2 .
$$

By Clifford's Theorem, $X \mid\left\langle N_{L}(P), A Z(G)\right\rangle$ has an invariant space of dimension less than or equal to $(p-1) / 2$. As this space is invariant for $N_{L}(P)$, it is invariant for $L$ and for $\langle L, A\rangle$.

LeMmA 7. Let $\Pi$ be a set of primes, all of which are greater than $n$. Let $G$ be a finite П-group with a faithful representation $X$ of degree $n$. Then $G$ is abelian.

Proof. Degrees of irreducible constituents of $X$ divide $|G|=|G|_{\Pi}$ and are no larger than $n$. Such degrees are 1 , and $G$ is abelian.

LEMMA 8. Let $G$ have a faithful representation $X$. Let $K$ be a normal subgroup of $G$ with $X \mid K$ irreducible. Let $x \in G$ have order $p$, an odd prime not dividing $|K|$. Then there exists $\gamma \in C$ with $\gamma^{p}=1$ and the primitive pth roots of 1 appearing equally often as eigenvalues of $\gamma X(x)$.

Proof. There are $p$ extensions of $X \mid K$ to $\langle K, x\rangle$. Let $Y$ be one of these. Then they are all of the form $Y \otimes A^{i}$ where $A$ is a faithful linear representation of $\langle K, x\rangle / K$. Since the character of $X$ does not vanish on $x(\operatorname{as} \operatorname{deg} X \not \equiv 0(\bmod p))$ and since the Galois group $\langle\sigma\rangle$ of the $p$ th roots of 1 permutes these $p$ representatives $Y \otimes A^{i}$ it follows that exactly one of them has a rational character, since $Y \otimes A^{i}=\sigma\left(Y \otimes A^{i}\right)=\left(Y \otimes A^{k}\right) \otimes A^{t i}=Y \otimes A^{k+t i}$ can be solved for $i$.

Lemma 9. Let $X$ be a faithful, irreducible, quasiprimitive representation of a finite group $G$ on an $n$-dimensional vector space $V$. Let $H$ be a subgroup of $G$ with $H / Z(H) \simeq P S L(2, p)$ for $p$ a prime greater than four. Suppose that $\operatorname{dim} C_{V}(H)$ $=n-(p-1) / 2$. Then $\operatorname{dim} C_{V}(H) \leqq 1$.

Proof. By Lemma 3, we may replace $H$ by $H^{\prime}$ with $H \simeq(P) S L(2, p)$. Assume that $C_{V}(H)>1$. There are two overlapping cases:

Case $1 .(p-1) / 2$ is even. In this case $H \simeq S L(2, p)$. Let $P=\langle x\rangle$ be a $p$-Sylow subgroup of $H$. By [13] we may write $X$ in matrix form in the ring of local integers of some algebraic number field for a prime ideal dividing (2). As $(2,|P|)=1$, by 
[13] and [10], we may further take $X \mid P$ to be diagonal. Let $z$ be the involution in $Z(H)$. By Lemma $2, X(x)$ has $n-(p-1) / 2$ eigenvalues 1 and $(p-1) / 2$ distinct eigenvalues $\varepsilon_{1}, \ldots, \varepsilon_{(p-1) / 2}$ unequal to 1 . We may write

$$
X(x)=\operatorname{diag}\left(1, \ldots, 1, \varepsilon_{1}, \ldots, \varepsilon_{(p-1) / 2}\right) .
$$

As $z \in C(x)$ and $C_{V}(P)=C_{V}(H) \subset C_{V}(z)$, there exist $\gamma_{i}$ with

$$
X(z)=\operatorname{diag}\left(1, \ldots, 1, \gamma_{1}, \ldots, \gamma_{(p-1) / 2}\right) .
$$

Let $Y$ be the modular representation obtained by taking coefficients in $X$ modulo the prime ideal dividing (2). Then $z \in K$, the kernel of $Y$. By [3], $K$ is a two group. By quasiprimitivity, we may change coordinates to write $X(K)=U(K) \otimes I_{m}$ for some irreducible representation $U$ of $K$ and some integer $m$. By [8, Satz 3], there exist functions $V$ and $W$ from $G$ to $G L(n / m, C)$ and $G L(m, C)$ respectively with $X(g)=V(g) \otimes W(g)$ for all $g \in G$. We may also take $V(x)$ to have order $p$. Then $V(x)$ normalizes $U(K)$. By Lemma $8, V(x)$ is scalar or has as many as $(p-1)$ distinct eigenvalues. As $X(x)$ has only $(p+1) / 2$ distinct eigenvalues, $V(x)$ is scalar. As $\varepsilon_{1}$ occurs only once as an eigenvalue of $X(x), \operatorname{dim} V=1$. Then $X(z)$ is scalar, a contradiction.

Case 2. Here $(p-1)$ is not a power of 2 . In this case, there exists $q$, an odd prime dividing $p-1$. Let $P=\langle x\rangle$ be a $p$-Sylow subgroup of $H$. Then there exists $y$ of order $q$ in $N_{H}(P)$. The normal subgroup $P^{G}$ generated by $P$ contains $H$. If it is reducible, then some constituent of $X \mid P^{G}$ has $H$ in the kernel, contrary to quasiprimitivity. Conjugates of $X(x)$ cannot permute spaces of imprimitivity nontrivially, for that would imply that $\mid$ trace $X(x) \mid \leqq n-p$. Therefore, $X \mid P^{G}$ is primitive. We may replace $G$ by $P^{G}$ and assume $G=P^{G}$. Then $\langle y\rangle^{G} \supset H,\langle y\rangle^{G} \supset H^{G} \supset P^{G}=G$, and $G=\langle y\rangle^{G}$. Write $X \mid H=X_{1} \oplus X_{2}$ where $H$ is in the kernel of $X_{1}$ and $\operatorname{deg} X_{2}$ $=(p-1) / 2$. The constituents of $X_{2} \mid P$ are distinct and nonprincipal. Also, $y$ fixes only principal characters of $P$. Therefore, $X_{2}(y)=0$ and the eigenvalue 1 occurs $(p-1) / 2 q$ times in $X_{2}(y)$ and $n-((p-1) / 2-(p-1) / 2 q)$ times in $X(y)$. If $u$ is any conjugate of $y$ in $G$ and $H_{u}=\left\langle H, u^{-1} H u\right\rangle$, then

$$
\begin{aligned}
n-\operatorname{dim} C_{V}\left(H_{u}\right) & \leqq n-\operatorname{dim} C_{V}(\langle H, u\rangle) \\
& =n-\operatorname{dim} C_{V}(H) \cap C_{V}(u) \leqq n-\operatorname{dim} C_{V}(H)+n-\operatorname{dim} C_{V}(u) \\
& =(p-1) / 2+(p-1) / 2-(p-1) / 2 q<p-1 .
\end{aligned}
$$

As $(p-1) / 2+(p-1) / 2+\operatorname{dim} C_{V}\left(H_{u}\right)>n$, by [6], $X \mid H_{u}$ has at most one constituent, say $X_{u}$ acting on the subspace $V_{u}$, with $i_{p}\left(X_{u}\left(H_{u}\right)\right) \neq 1$. By [5], $i_{p}\left(X_{u}\left(H_{u}\right)\right) \leqq p$. Since $i_{p}(H)=p$, by Lemma 1 , such an $X_{u}$ exists, $i_{p}\left(X_{u}(H)\right)=i_{p}\left(X_{u}\left(u^{-1} H u\right)\right)=p$, and $X_{u}$ contains the nonprincipal constituent of $X \mid H$ and the nonprincipal constituent of $X \mid u^{-1} H u$. Let $Y_{u}$ be a complement to $X_{u}$ for $X \mid H_{u}$. Then $X \mid H_{u}=Y_{u} \oplus X_{u}$. As $H$ and $u^{-1} H u$ are in the kernel of $Y_{u}, H_{u}$ is in the kernel of $Y_{u}$ and $V=C_{V}\left(H_{u}\right) \oplus V_{u}$. By Lemma 4 applied to $X_{u}$ and $H \subset H_{u}$ :

$$
\operatorname{deg} X_{u}=(p \pm 1) / 2 \text { and } \operatorname{dim} C_{V}\left(H_{u}\right)=n-(p \pm 1) / 2
$$


If $\operatorname{deg} X_{u}=(p-1) / 2$, then $u^{-1} C_{V}(H)=C_{V}\left(u^{-1} H u\right)=C_{V}\left(H_{u}\right)=C_{V}(H)$. As $C_{V}(H)$ is not invariant for $G=\langle y\rangle^{G}$ we may find $u_{0}$ conjugate to $y$ with $\operatorname{deg} X_{u_{0}}=(p+1) / 2$ (actually, $\operatorname{deg} X_{u_{0}}=(p+1) / 2$ is impossible by [12], but we go on, anyway) and $\left.\operatorname{dim} C_{V}\left(H_{u_{0}}\right)=n-(p+1) / 2\right\rangle 0$. Since $G=H^{G}$ and $G=\langle y\rangle^{G}, G=\left\langle v^{-1} H v\right| v=u_{1} \cdots u_{r}$ where $u_{i}$ is a conjugate of $y$ in $G$ for $\left.i=1, \ldots, r\right\rangle$. As $C_{V}\left(H_{u_{0}}\right)$ is not invariant under $X(G)$, we may find $v=u_{1} \cdots u_{r}, u_{i}$ conjugate to $y$ for $i=1, \ldots, r$, with $C_{V}\left(H_{u_{0}}\right)$ not invariant under $v^{-1} H v$. Then $C_{V}\left(H_{u_{0}}\right) \notin C_{V}\left(v^{-1} H v\right)$ and $C_{V}\left(\left\langle v^{-1} H v, H_{u_{0}}\right\rangle\right) \neq$ $C_{V}\left(H_{u_{0}}\right)$. Take $v$ so that $C_{V}\left(\left\langle v^{-1} H v, H_{u_{0}}\right\rangle\right) \neq C_{V}\left(H_{u_{0}}\right)$ and $r$ is minimal. Then $r \geqq 1$. Define $w=v u_{r}^{-1}=u_{1} \cdots u_{r-1}$. Then $C_{V}\left(\left\langle w^{-1} H w, H_{u_{0}}\right\rangle\right)=C_{V}\left(H_{u_{0}}\right)$. Letting $w^{-1} H w$ play the role of $H$ and $u_{r}$ play the role of $u$, we have

$$
C_{V}\left(\left\langle w^{-1} H w, v^{-1} H v\right\rangle\right)=C_{V}\left(\left\langle w^{-1} H w, u_{r}^{-1}\left(w^{-1} H w\right) u_{r}\right\rangle\right)=n-(p \pm 1) / 2 .
$$

As $C_{V}\left(\left\langle w^{-1} H w, v^{-1} H v\right\rangle\right) \subset C_{V}\left(w^{-1} H w\right)$ and $C_{V}\left(H_{u_{0}}\right) \subset C_{V}\left(w^{-1} H w\right)$,

$\operatorname{dim} C_{V}\left(\left\langle v^{-1} H v, H_{u_{0}}\right\rangle\right) \geqq \operatorname{dim} C_{V}\left(\left\langle w^{-1} H w, v^{-1} H v\right\rangle\right) \cap C_{V}\left(H_{u_{0}}\right)$

$$
\begin{aligned}
& \geqq \operatorname{dim} C_{V}\left(\left\langle w^{-1} H w, v^{-1} H v\right\rangle\right)+\operatorname{dim} C_{V}\left(H_{u_{0}}\right)-\operatorname{dim} C_{V}\left(w^{-1} H w\right) \\
& \geqq n-(p+1) / 2+n-(p+1) / 2-(n-(p-1) / 2)>n-(p-1) .
\end{aligned}
$$

As with $X_{u}$, by [6], $X \mid\left\langle v^{-1} H v, H_{u_{0}}\right\rangle$ has exactly one irreducible constituent $W$ acting on $U_{w}$ with $i_{p}\left(W\left(\left\langle v^{-1} H v, H_{u_{0}}\right\rangle\right)\right) \neq 1$. By [5], $i_{p}\left(W\left(\left\langle v^{-1} H v, H_{u_{0}}\right\rangle\right)\right)=p$. This constituent $W$ contains the nonprincipal constituents of $X \mid v^{-1} H v$ and $X \mid H_{u_{0}}$, so $V=C_{V}\left(\left\langle v^{-1} H v, H_{u_{0}}\right\rangle\right) \oplus U_{w}$. By Lemma 4 applied to $W$ and $H_{u_{0}} \subset\left\langle v^{-1} H v, H_{u_{0}}\right\rangle$ : $\operatorname{deg} W=(p+1) / 2, \operatorname{dim} C_{V}\left(\left\langle v^{-1} H v, H_{u_{0}}\right\rangle\right)=n-(p+1) / 2=\operatorname{dim} C_{V}\left(H_{u_{0}}\right)$, and

$$
C_{V}\left(\left\langle v^{-1} H v, H_{u_{0}}\right\rangle\right)=C_{V}\left(H_{u_{0}}\right)
$$

a contradiction.

Proof of the theorem. We use induction on $n=\operatorname{deg} X$ and assume that the finite group $G$ with representation $X$ is a counterexample to the theorem with $n$ minimal for a fixed set of primes, $\Pi$. By Lemma 7 we may let $H$ be an abelian $\Pi$-Sylow subgroup of $G$.

(A). If $L \subset G$ and $X \mid L$ is reducible, then $L$ satisfies the conclusion of the theorem. In particular $X \mid G$ is irreducible.

Proof. Let $L$ contradict (A) and $X \mid L=Y_{1} \oplus Y_{2}$. Then $\operatorname{deg} Y_{i}<n \leqq p-1$ for all $p \in \Pi$ and $i=1,2$. By the minimality of $n, i_{\Pi}\left(Y_{i}(L)\right)$ is not composite for $i=1,2$. If for $i=1$ or $2, \operatorname{deg} Y_{i}<(p-1) / 2$ for all $p \in \Pi$, then by [6], $i_{\Pi}\left(Y_{i}(L)\right)=1$, and by Lemma $1, i_{\Pi}(L)$ is not composite. Therefore, $\operatorname{deg} Y_{1}=n / 2=p-1$ for some $p \in \Pi$ and $i_{\Pi}\left(Y_{i}(L)\right)=p$ for $i=1,2$. Then $i_{\Pi}(L)=p$, or Lemma 5 applied to $L$ gives the conclusion.

(B). We may choose $X$ and $G$ so that $X$ is unimodular. Then $H \cap Z(G)=\langle 1\rangle$.

Proof. By [1], we may find a finite group $L$ with a faithful, unimodular representation $Y$ of dimension $n$ with $X(G) Z G L(n, C)=Y(L) Z G L(n, C)$. Then $Y$ is irreducible. 
Now $G$ has a $\Pi$-Sylow subgroup and $L / Z(L) \simeq G / Z(G)$ has a $\Pi$-Sylow subgroup. Let $U \supset Z(L)$ and $U Z(L)$ be a $\Pi$-Sylow subgroup of $L / Z(L)$. As $[Z(L)]_{\Pi^{\prime}}$ is a normal $\Pi$ '-Sylow subgroup of $U Z(L)$, by Schur-Zassenhaus, $U Z(L)$ has a $\Pi$-Sylow subgroup, and this is a $\Pi$-Sylow subgroup for $L$. Now $i_{\Pi}(G)=i_{\Pi}(G / Z(G))=i_{\Pi}(L / Z(L))$ $=i_{\Pi}(L)$ is composite. If $V=V_{1} \oplus V_{2}$ gives spaces of imprimitivity for $Y(L)$, then $X(G)$ has the same spaces of imprimitivity and a normal subgroup $K$ of index 1 or 2 leaves $V_{1}$ and $V_{2}$ invariant. As $O_{\Pi}(K)$ is characteristic in $K$ and $K \triangleleft G, O_{\Pi}(K)$ $\subset O_{\Pi}(G)$. Then $G$ satisfies whichever alternative of the conclusion of the theorem that $K$ satisfies by (A). Therefore, $Y$ and $L$ are a counterexample to the theorem and may be used to replace $X$ and $G$. Then $X$ may be taken to be unimodular. Then if $x \in H \cap Z(G), X(x)=\gamma I_{n}$ where $\gamma^{n}=1$, and $\gamma$ must be 1 .

(C). We may further choose $G$ with $G=O^{\Pi^{\prime}}(G)=H^{G}$, and with $X$ being primitive.

Proof. Both $O^{p^{\prime}}(L)$ and $H^{G}$ are the subgroup of $G$ generated by all $\Pi$-elements. Also, $H \subset H^{G}$. If $i_{\Pi}\left(H^{G}\right)$ is not composite, then as $O_{\Pi}\left(H^{G}\right)$ is characteristic in $H^{G} \triangleleft G, O_{\Pi}\left(H^{G}\right) \subset O_{\Pi}(G)$, and $i_{\Pi}(G)$ is not composite, a contradiction. Suppose that $V=V_{1} \oplus V_{2}$ and $O_{\Pi}\left(H^{G}\right)$ is imprimitive on the $V_{i}, i=1,2$. As a subgroup of $H^{G}$ of index 2 contains all $\Pi$-elements of $H^{G}$ and, hence of $G$ and must equal $H^{G}$, it follows that $V_{1}$ and $V_{2}$ are invariant for $H^{G}$. As $i_{\Pi}\left(H^{G}\right)$ is composite, by (A), $X\left(H^{G}\right)$ satisfied II of the theorem. As $V_{1}$ and $V_{2}$ are the unique invariant subspaces of dimension $n / 2$ for $H^{G} \triangleleft G, G$ is imprimitive on the $V_{i}, i=1,2$; and satisfies II of the theorem, a contradiction. As $O^{\Pi^{\prime}}\left(O^{\Pi^{\prime}}(G)\right)$ contains all $\Pi$-elements of $O^{\Pi^{\prime}}(G)$ and of $G, O^{\Pi^{\prime}}\left(O^{\Pi^{\prime}}(G)\right)=O^{\Pi^{\prime}}(G)$. As $O^{\Pi^{\prime}}(G)$ is a contradiction to the theorem, we may replace $G$ by $O^{\Pi^{\prime}}(G)$. Then we have $G=O^{\Pi^{\prime}}(G)=H^{G}$. If $V_{1}, \ldots, V_{m}$ form spaces of imprimitivity for $G$, then $m \leqq n<p$ for all $p \in \Pi$ and $\Pi$-elements must fix the $V_{i}$. Then $G=H^{G}$ fixes the $V_{i}$. Then, by (A), $m=1$.

(D). If $x$ is a $\Pi$-element with an eigenvalue occurring more than $n / 2$ times in $X(x)$, then $x=1$.

Proof. Otherwise, we may take $x \in H$ of order $p$, a prime, with $X(x)$ having eigenvalues $\alpha, \alpha, \ldots, \alpha, \alpha_{1}, \ldots, \alpha_{m}, m<n / 2$. If $\langle x\rangle^{G}$ is abelian, then by quasiprimitivity, (C), $X \mid\langle x\rangle^{G}$ has identical linear constituents and $\langle x\rangle^{G} \subset Z(G)$, a contradiction. Therefore, we may find $y$, a conjugate of $x$ not in $C(x)$. Let $K=\langle x, y\rangle$. By Lemma $7, K$ is not a $p$-group, and $i_{p}(K) \neq 1$. Therefore, by Lemma 1 , there exists an irreducible constituent $Y$ of $X \mid K$ with $i_{p}(Y(K))>1$. Now, $C_{V}\left(\alpha^{-1} x\right)$ $\cap C_{V}\left(\alpha^{-1} y\right)$ is a sum of linear constituents for $X \mid K$. Also,

$$
\begin{aligned}
& n-\operatorname{dim} C_{V}\left(\alpha^{-1} x\right) \cap C_{V}\left(\alpha^{-1} y\right) \\
& \leqq n-\operatorname{dim} C_{V}\left(\alpha^{-1} x\right)+n-\operatorname{dim} C_{V}\left(\alpha^{-1} y\right) \leqq 2 n-2 m<n .
\end{aligned}
$$

Therefore, $\operatorname{deg} Y<n$ and by minimality of $n, i_{p}(Y(K))=p$. As $Y(x) \notin Z(Y(K))$, by Lemma 2, $Y(x)$ has distinct eigenvalues. Let $d$ be the number of $\alpha_{1}, \ldots, \alpha_{m}$ occurring as eigenvalues in $Y(x)$. Then

$$
n / 2 \leqq(p-1) / 2 \leqq \operatorname{deg} Y=\operatorname{var} Y(x) \leqq 1+d \leqq 1+m<1+(n / 2) .
$$


Then $(p-1) / 2=\operatorname{deg} Y=\operatorname{var} Y(x)=1+d=1+m$. Replacing $x$ by $y$ above, we see that a complement $U$ to $Y$ for $X \mid K$ has $U(x)=U(y)=\alpha I_{n-(p-1) / 2}$. By Lemma 2 applied to $Y(K)$, there exists $u$ of order $p$ in $K^{\prime}$ with $Y(x) Y\left(u^{-1}\right) \in Z(Y(K))$ and $Y(u)$ having $(p-1) / 2$ distinct eigenvalues, all unequal to 1 . As $u \in K^{\prime}, U(u)=$ $I_{n-(p-1) / 2}$. Then $\operatorname{var} X(u)=1+(p-1) / 2$. As $Y\left(x u^{-1}\right)$ and $U\left(x u^{-1}\right)$ are both scalar of order dividing $p$, var $Y\left(x u^{-1}\right) \leqq 2$. As $X$ is primitive and $p \geqq 7$, by [1], $X\left(x u^{-1}\right)$ is scalar. Then

$$
\operatorname{var} X(x)=\operatorname{var} X(u)=1+(p-1) / 2 \geqq 1+n / 2>1+m,
$$

a contradiction.

(E). If $x$ is a nonidentity $\Pi$-element, then $i_{\Pi}(C(x))=1$.

Proof. Otherwise, by Lemma $1, X \mid C(x)$ has an irreducible constituent $Y$ with $i_{\Pi}(Y(C(x))) \neq 1$. By [6], deg $Y \geqq(p-1) / 2$ for some $p \in \Pi$. Then some eigenvalue occurs in $X(x)$ with multiplicity $m \geqq(p-1) / 2 \geqq n / 2$. By (D), $m=(p-1) / 2=n / 2$ and $(n+1) \in \Pi$. Let $U$ be a complementary constituent to $Y$ for $X \mid C(x)$. If $U(C(x))$ does not have a normal abelian $p$-Sylow subgroup, then by [6], $U$ is irreducible, var $X(x) \leqq 2$, var $X(x)=1$ by [1] and primitivity, $x \in Z(G)$, and by (B) $x=1$, a contradiction. Therefore, $\left(O^{p^{\prime}}(C(x))\right)^{\prime}$ is in the kernel of $U$. By Lemma 3,

$$
Y\left(\left(O^{p^{\prime}}(C(x))\right)^{\prime}\right) \simeq(P) S L(2, p) .
$$

Then $\left(O^{p^{\prime}}(C(x))\right)^{\prime}$ contradicts Lemma 9.

(F). $H$ is a trivial intersection set (T. I. S.) in $G$.

Proof. Let $x \in H^{\#} \cap g^{-1} H g$. Then $H, g^{-1} H g \in C(x)$. By $(\mathrm{E}), i_{\mathrm{II}}(C(x))=1$. Then $H=O_{\mathrm{II}}(C(x))=g^{-1} \mathrm{Hg}$.

(G). If $K \subset G$ and $O_{\mathrm{\Pi}}(K) \neq\langle 1\rangle$, then $i_{\mathrm{\Pi}}(K)=1$.

Proof. Let $K$ contradict (G). If $x$ is a $\Pi$-element of $K$, then $\left\langle x, O_{\Pi}(K)\right\rangle$ is a $\Pi$-group, and by Lemma 7, $x \in C\left(O_{\Pi}(K)\right)$. Therefore, $O^{\Pi^{\prime}}(K) \subset C\left(O_{\Pi}(K)\right) \subset C(y)$ for some nonidentity $\Pi$-element $y$ in $O_{\Pi}(K)$. By (E) and Lemma $1, i_{\mathrm{II}}\left(O^{\Pi^{\prime}}(K)\right)=1$. Then $O_{\Pi}\left(O^{\prime}(K)\right)$ is a normal $\Pi$-Sylow subgroup of $K$.

(H). If $x \notin Z(G)$, then $i_{\Pi}(C(x))=1$.

Proof. As $C\left([x]_{\mathrm{II}}\right) \supset C(x)$, by (E) and Lemma 1, we may assume that $x$ is a $\Pi^{\prime}$-element contradicting $(\mathrm{H})$. By $(\mathrm{G}), O_{\Pi}(C(x))=\langle 1\rangle$. By (A) applied to $C(x)$, $i_{\Pi}(C(x))=p$ for some $p \in \Pi$; otherwise, the II of the theorem gives a subgroup contradicting Lemma 9. Therefore, $|C(x)|_{\Pi}=p$. Replacing $x$ by a conjugate of $x$ there exists a $p$-Sylow subgroup $P=\langle y\rangle$ of $C(x)$ contained in $H$.

If $X \mid C(x)$ has two constituents $X_{1}$ and $X_{2}$ with $i_{p}\left(X_{i}(C(x))\right)=p$ for $i=1,2$, then, by [6], $X \mid C(x)=X_{1} \oplus X_{2}$ with $p=(n+1) \in \Pi, \operatorname{deg} X_{i}=(p-1) / 2$, and $X_{i}(C(x)) / Z\left(X_{i}(C(x))\right) \simeq P S L(2, p)$ for $i=1,2$. By Lemma 3 , there is a subgroup $K$ of $C(x)^{\prime}$ with $K \simeq(P) S L(2, p)$, and $X_{i}$ are either the two distinct $(p-1) / 2$ dimensional representations of $(P) S L(2, p)$ or are identical. In the first case, $X(y)$ has mutually distinct eigenvalues and $C(y)$ is abelian. Then $\langle H, x\rangle \subset C(y)$ and $H \subset C(x)$ contrary to $|C(x)|_{\mathrm{II}}=p$. In the second case, we may change coordinates to write 
$X \mid K=X_{1} \otimes I_{2}$ and apply Lemma 6 with $A=H, L=K$ to conclude that $X \mid\langle H, K\rangle$ is reducible. We may apply (A) to $\langle H, K\rangle$. As II of the theorem gives a subgroup contradicting Lemma $9, i_{\Pi}(\langle H, K\rangle)$ is not composite, $O_{\Pi}(\langle H, K\rangle) \neq\langle 1\rangle$. By (G), $i_{\Pi}(\langle H, K\rangle)=1$. Then $p \leqq i_{\Pi}(K) \leqq i_{\Pi}(\langle H, K\rangle)=1$, a contradiction.

Therefore, $X \mid C(x)$ has exactly one irreducible constituent, say $Y$ acting on the subspace $S$, with $i_{p}(Y(C(x))) \neq 1$. Let $U$, acting on the subspace $T$, be a complement to $Y$ for $X \mid C(x)$. Let $K=\left(O^{p^{\prime}}(C(x))\right)^{\prime}$. Then $K \subset$ ker $U$. By Lemma 2, there exists $u$ of order $p$ in $K$ with $Y(u)$ having $m \geqq(p-1) / 2$ eigenvalues unequal to 1. As $|C(x)|_{p}$ $=p$, we may choose $u$ to be $y$. Let $W=\sum_{\beta \neq 1} C_{V}\left(\beta^{-1} y\right)$. Then $W \subset S$. Also, $m=$ $\operatorname{dim} W$, and $m \geqq(p-1) / 2$. Furthermore, $X(x)$ acts as a scalar on $S$, and, therefore, also on $W$. As $\langle H, x\rangle \subset C(y), W$ is invariant under $\langle H, x\rangle$. For any $h \in H, X((h, x))$ acts as a scalar on $W$ and $X((h, x))$ has at least $m$ eigenvalues equal to 1 . As $H$ is a T. I. S. and $y \in H \cap C(x), x \in N(H)$ and $(h, x) \in H$. By (D), $(p-1) / 2 \leqq m \leqq n / 2$ or $(h, x)=1$. Therefore, $2 m+1=p=(n+1) \in \Pi$ or $H \subset C(x)$. As $|C(x)|_{\Pi}=p, 2 m+1$ $=p=(n+1) \in \Pi$. Then $\operatorname{deg} Y=(p+1) / 2$, otherwise, $K$ contradicts Lemma 9. Now $T \oplus S=V=C_{V}(y) \oplus W$ with $C_{V}(y)$ and $W$ invariant under $\langle H, x\rangle \subset C(y)$. By Lemma $2, Y \mid K$ is irreducible. Let $h$ be any element of $H$. Then $C_{V}\left(h^{-1} K h\right)=$ $h^{-1} C_{V}(K) \subset h^{-1}\left(C_{V}(y)\right)=C_{V}(y)$. Then

$$
\begin{aligned}
\operatorname{dim} C_{V} & \left.\left\langle K, h^{-1} K h\right\rangle\right) \\
& =\operatorname{dim} C_{V}(K) \cap C_{V}\left(h^{-1} K h\right) \geqq \operatorname{dim} C_{V}(K)+\operatorname{dim} C_{V}\left(h^{-1} K h\right)-\operatorname{dim} C_{V}(y) \\
& =(p-3) / 2+(p-3) / 2-(p-1) / 2=(p-5) / 2>0 .
\end{aligned}
$$

Then by [6] and Lemma $1, X \mid\left\langle K, h^{-1} K h\right\rangle$ has at most one constituent $R$ with $i_{p}\left(R\left(\left\langle K, h^{-1} K h\right\rangle\right)\right) \neq 1$. The constituent $R$ must contain the constituent $Y$ for $R \mid K$. As $\operatorname{deg} R<n$, by minimality of $n, i_{p}\left(R\left(\left\langle K, h^{-1} K h\right\rangle\right)\right)=p$. By Lemma 4 applied to $R$ and $K \subset\left\langle K, h^{-1} K h\right\rangle, \operatorname{deg} R=\operatorname{deg} Y$. Then $S$ is invariant under $X(h)$. As $X(x)$ is scalar on $S, S \subset C_{V}((h, x))$ and by (D), $(h, x)=1$. Then $H \subset C(x)$ a contradiction.

(I). Let $N_{0}=\left\{\bigcup_{1 \neq y \in H} C(y)\right\}-Z(G)$. Then if $g \notin N(H), N_{0} \cap g^{-1} N_{0} g$ is empty.

Proof. Let $x \in N_{0} \cap g^{-1} N_{0} g$. Then there exist $h, k \neq 1, h, k \in H$ with $h, g^{-1} k g$ $\in C(x)$. By $(\mathrm{H}), i_{\mathrm{I}}(C(x))=1$, so $h, g^{-1} \mathrm{~kg} \in O_{\mathrm{\Pi}}(C(x))$. By Lemma 7, $O_{\mathrm{I}}(C(x)) \subset C(h)$, $C\left(g^{-1} k g\right)$. As $H$ is a T. I. S., $O_{\Pi}(C(x)) \subset N(H), N\left(g^{-1} H g\right)$. Then $\left\langle O_{\Pi}(C(x)), H\right\rangle$, $\left\langle O_{\Pi}(C(x)), g^{-1} H g\right\rangle$ are $\Pi$-groups, and $\langle h\rangle \subset O_{\Pi}(C(x)) \subset H \cap g^{-1} H g$. Then $H=g^{-1} H g$.

(J). By (C) and (I), $H \subset G$ satisfies the hypothesis of Lemma 4.2 of [5], by which $n+1>|H|^{1 / 2} \geqq p$ for some $p$ in $\Pi$, a contradiction.

\section{BIBLIOGRAPHY}

1. H. F. Blichfeldt, Finite collineation groups, University of Chicago Press, Chicago, Ill., 1917.

2. R. Brauer, On groups whose order contains a prime to the first power. I, II, Amer. J. Math. 64 (1942), 401-440. MR 4, 1; MR 4, 2.

3. - Uber endlichen lineare Gruppen von Primzahlgrad, Math. Ann. 169 (1967), 73-96 MR 34 \#5913. 
4. C. Curtis and I. Reiner, Representation theory of finite groups and associative algebras, Pure and Appl. Math., vol. XI, Interscience, New York, 1962. MR 26 \#2519.

5. W. Feit, Groups which have a faithful representation of degree less than $p-1$, Trans. Amer. Math. Soc. 112 (1964), 287-303. MR 28 \#5110.

6. W. Feit and J. G. Thompson, Groups which have a faithful representation of degree less than ( $p-1 / 2)$, Pacific J. Math. 11 (1961), 1257-1262. MR 24 \#A3207.

7. G. Glauberman, Correspondences of characters for relatively prime operation groups, Canad. J. Math. 20 (1968), 1465-1488. MR 38 \#1189.

8. B. Huppert, Lineare auflösbare Gruppen, Math. Z. 67 (1957), 479-518. MR 19, 729.

9. N. Itô, On a theorem of H. F. Blichfeldt, Nagoya Math. J. 5 (1953), 75-77. MR 14, 842.

10. I. Schur, Uber eine Klasse von endlichen Gruppe linearer Substitutionen, S.-B. Preuss. Akad. Wiss. (1905), 77-91.

11. - Untersuchungen über die Darstellung der endlichen Gruppen durch gebrochene lineare Substitutionen, J. Reine Angew. Math. 132 (1907), 85-137.

12. H. F. Tuan, On groups whose orders contain a prime to the first power, Ann. of Math. (2) 45 (1944), 110-140. MR 5, 143.

13. H. Zassenhaus, Neuer Beweis der Endlichkeit der Klassenzahl bei unimodularer Aquivalenz endlicher ganzzahliger Substitutionsgruppen, Abh. Math. Sem. Univ. Hamburg 12 (1938), 276-288.

THE RAND CORPORATION,

Santa Monica, California 90406 